\title{
Users Integrity Constraints in SOLAP Systems. Application in Agroforestry
}

\author{
Abdallah Bensalloua Charef*, Hamdadou Djamila \\ Computer Science Laboratory of Oran (LIO) - University of Oran 1 Ahmed Benbella, Oran (Algeria) \\ Received 6 August 2017 | Accepted 16 December 2017 | Published 26 January 2018
}

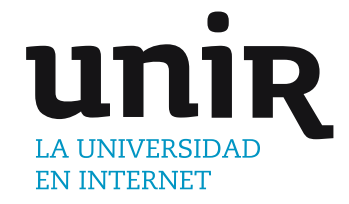

KEYWORDS

Agroforestry, Integrity

Constraints, SOLAP,

Spatial Data Warehouse, Spatial Decision Support System, Spatial

Multidimensional

Analysis, UML

Multidimensional

Modeling. we define inter-facts ICs in this case. In order to validate our proposition, we propose the multidimensional modeling by UML profile to support constellation schema of a hypercube with several fact tables related to subjects of analysis in forestry management. Then, we propose implementation of some ICs related to users of such a system.
DOI: $10.9781 /$ ijimai.2018.01.003

\section{INTRODUCTION}

PATIAL On-Line Analytical Processing(SOLAP)and Spatial Data $\checkmark$ Warehouses (SDWs) are decision support technologies that allow spatial and multidimensional analysis of multisource data. Basing on the multidimensional model, these technologies allow a multidimensional representation of data in hypercubes (datacubes). The hypercubes are essentially based on facts and dimensions concepts. The facts tables are analysis subjects which are described by numerical, textual or spatial measures. The dimensions define the analyzing axis. They can be organized in hierarchies of several levels. This allows the visualization of the measures at different levels of detail.

The data quality in the spatial hypercubes is important since this data is used as a basis for decision-making. In fact, poor data quality could lead to poor decision making. Without considering data quality in spatial hypercubes, it may provide unreliable results. As a result, integrity constraints (ICs) become very important to improve the logical consistency of any database, which increase data quality.

In transactional spatial databases, spatial integrity constraints are defined along the database conceptual models to preserve spatial data quality [1], [2]. However, in analytical process other kind of integrity constraints must be considered for maintaining consistent analysis using the hypercube. It is related to the manner of navigating and querying data with consideration of aggregation rules, hierarchies and other concepts of the multidimensional models.

In the database community, some research works study integrity

\footnotetext{
* Corresponding author.

E-mail address: charef_bensalloua@yahoo.fr
}

constraints for non-spatial datacubes [3], [4]. However, for studying integrity constraints for spatial datacube, its specific characteristic related especially to spatial data features should be considered. Therefore, the objective of this paper is to propose a system for the implementation of user integrity constraints in SOLAP namely "UICSOLAP". It takes into account fundamental considerations about users querying and aggregating in spatial OLAP. In addition, other data integrity constraints are implemented in order to obtain efficient analysis within the decision-making process.

Section 2 reviews a state of the art related to quality management in SDW and SOLAP in one hand. In the other hand, some researches in the field of forestry management are cited. In Section 3 our contribution is stated. Section 4 discusses the integrity constraints from spatial databases to spatial datacubes. In Section 5 the proposed system namely "UIC-SOLAP" for user integrity constraints in SOLAP is depicted while the process adopted by it, is presented in Section 6. Section 7 explains the case study related to an implementation of Spatial Data Warehouse for forest management. In Section 8, obtained results are discussed related to the application of the "UIC-SOLAP" system with SOLAP prototype developed for forestry management. Finally, Section 9 concludes and draws more research directions for the implementation of the integrity constraints and data quality in spatial hypercubes.

\section{STATE OF THE ART}

Quality management in SDW and SOLAP systems is an important research issue. Indeed, precision in SDW was addressed in [5] and [6] which provide logic models and an indexing technique for storing and querying vague spatial data. 
In [7] authors have studied the problem of completeness in classical DW. They proposed solution taking into account missing values in hierarchies. Inconsistencies which refer to the existence of logical contradictions in SDW can be also controlled by ICs. As mentioned in [8], ICs expression on conceptual models is essential for taking into account all quality rules. For this aim, [9] proposes ad-hoc multidimensional conceptual models allowing the expression of some data ICs using logic predicates. In [10], authors propose an extension of the Entity-Relationship (ER) model for the design of spatio-temporal data warehouses. They define a set of ad hoc pictograms to express ICs on spatial data (topological relations between spatial members). Authors in [11] propose a UML profile for DWs, but they consider only a very small number of data ICs. Aggregation problems are presented in [12] by defining simple schema constraints with UML multiplicities. In [13], the author concludes that several languages for the specification of ICs in spatial databases have been proposed but they are not efficient for defining ICs in spatial hypercubes. Indeed, the author presents a formal language for the specification of integrity constraints in the conceptual model of spatial hypercubes. This language is based on controlled natural languages as well as natural hybrid languages with pictograms. In [14], authors study complex structural aggregation constraints. Basing on a UML(Unified Modeling Language) model, [8] shows that the Spatial OCL (Object Constraints Language) which is an OCL extension for spatial data, allows the definition of a large number of ICs on spatial data. Data modeling languages can express only a very limited number of integrity constraints as for example the cardinalities of the relations between classes of objects. For the expression of IC, Many works use non-standard languages, namely logical, natural, visual or hybrid. Others prefer OCL because it integrates easily with UML. Therefore, it is necessary to use languages dedicated for the ICs specification that are interoperable with the language of definition of the data structures. In [15], the author presents a method for the conceptual modeling of the SOLAP ICs after having introduced the main concepts on which this method is based namely the UML profiles and Spatial OCL.

In the field of natural resources specifically in the forest management and preserving, researches published are relevant for consideration in forest environments as well as in social and economic anxieties. In [16], the author implements a participatory decision making in forest planning. Also, authors in [17], propose a Decision Support for forest management. Authors in [18] treat the problem of forest land use policies. In [19], authors integrate multiple criteria decision analysis in participatory forest planning. Authors in [20] propose a participatory multi-criteria assessment of forest planning policies in conflicting situations. An empirical study on voting power in participatory forest planning is proposed in [21]. For forest protection, authors in [22], propose software for forest fire detection and extinction.

\section{CONTRIBUtion}

In forestry, we notice the complexity of effective management of natural areas for ecological, economic and social purposes. Indeed, the use of SOLAP application for decision-making allows analyzing this domain in various axes of reflection. However, different kinds of heterogeneity may exist between the data sources (remote sensing, GIS applications ... etc.). As a result, several problems of inconsistency may emerge. In most studies, the discussed problems are related to the heterogeneity of manipulated data. So, few studies have addressed issues related to the end users when decision-making is related to many sub-domains.

We notice also, that, the same territory is usually, under the control of several actors with different anxieties namely territorial management, exploiting of forest products and even local collectivities with social anxieties. However, the use of SOLAP tool by different actors independently allows them to extract decisional indicators which are heterogeneous. In fact, different user's ICs will generate unsteadiness in the whole decision system.

To the best of our knowledge, no study has treated the formulation of users ICs when using a hypercube with multiple facts tables. Indeed, defining multiple user-defined ICs may generate semantic inconsistencies which affect the analytical quality of such a system.

The main objective of the current study is to propose a system for the implementation of user integrity constraints in SOLAP namely "UIC-SOLAP".

Our proposal integrates all defined Integrity Constraints which are related to the users of SOLAP system along different stages. Indeed, properties of objects in spatial data sources may be adjusted according to every kind of final user. Also, we must verify user queries and aggregations when requesting the SOLAP by the user.

In addition, Integrity Constraints must be defined to respect particular information of a user, so that only concerned data is accessible. These are concerned by the respect of Integrity Constraints and they are all integrated in our proposal namely "UIC-SOLAP".

Secondary objectives are also achieved in this paper. Indeed, we propose a classification of ICs related to the users of SOLAP systems. After that, we propose a multidimensional model for forest spatial data warehouse (SDW). For this aim, we propose a UML profile which allows considering the main properties of spatial and Multi Dimensional (MD) modeling. We integrate also, users ICs definition with formalization of users profiles for specifying visualization ICs according to their preferences. Also, we define inter-facts ICs within the same hypercube. Indeed, unlike the traditional OLAP world, Unified Dimensional Model (UDM), the model for data in Microsoft SQL Server Analysis Services (SSAS), allows to have multiple fact tables within a cube [23].

Then, we propose the exploitation of the UML profile to support "constellation schema" of the forestry Spatial Data Warehouse (SDW). This will help to build a hypercube with several fact tables related to subjects of analysis in forestry management.

Finally, we test our proposal using data concerning forest areas of Mostaganem in Algeria to apply our methodology for verifying the user's integrity constraints in such a system.

\section{INTEGRITy CONSTRAINTS FROM SPATIAL DATABASES to Spatial Datacubes}

Spatial Data Warehouses (SDWs) integrate, organize in a multidimensional way and store very large volumes of spatial and non-spatial data from multiple sources to support the decision-making process within an organization [24].

Spatial OLAP (SOLAP) systems are a category of software tools that allow interactive exploration based on a spatio-multidimensional approach at several levels of detail of the SDW [25]. They extend and enrich the OLAP systems by new concepts and operators which allow effectuating spatial analyzes. Consequently, they allow the visualization of the results of analytical queries in the form of tables, charts and maps.

SOLAP systems are based on data stored in SDWs. As a result, SOLAP analysis quality includes spatial data quality which is the characteristic that distinguishes spatial datacubes from traditional ones.

Here, we must take into account the internal quality defined by its geometric and semantic accuracy; and the external quality which is related to the user needs or quality in the context of use. 
In the Spatial Databases, [26] classifies spatial ICs into three categories:

1. Topological ICs which include all geometric properties and relationships on data;

2. Semantic ICs which concern the meaning of geographical features and

3. User-defined ICs which are business rules in spatial databases.

However, in [13], the author notes some limitations when trying to apply these ICs to real applications.

In the Temporal Databases, a formal classification for temporal ICs is introduced in[27]. Indeed, ICs are categorized according to the transaction time (when a fact is stored in the database), valid time (when a fact was true in the reality), and both.

In the Spatiotemporal Databases static and transition ICs is integrated in[26] with the categories of spatial ICs. Here, other temporal ICs are involved. They restrict the possible lifecycle of objects like the IC that specifies the period of applicability of a rule.

The quality of SOLAP decision-making depends on the quality of the data stored and on the way in which the data is explored. Integrity constraints (ICs) are defined in [28] as assertions, typically defined in the conceptual model of an application, that are aimed at preventing the appearance of incorrect data in a database. In SOLAP system context, IC can be used for:

1. identifying erroneous data stored,

2. identifying incorrect analytical queries and

3. defining correct aggregation rules.

In analytical processing, a multidimensional model is based on concepts like dimension, hierarchy, level, member, fact, and measure. As a result, an integrity constraint specification must hold specific semantics supporting these concepts. Hence, it is more efficient to express spatial multidimensional integrity constraints referring to multidimensional elements. At the conceptual level, SDW ICs define conditions that data, metadata or analytical expressions are expected to satisfy. In [8], authors see that the definition of ICs at a conceptual level makes it possible to consider quality problems in the early stages of development. Indeed, they propose two new classifications of SDW ICs: (1) a classification oriented SDW concepts (Fact, Dimension, Aggregation, etc.) and (2) an implementation-oriented classification. The first category categorizes ICs according to the multidimensional elements that these ICs imply. The second group categorizes ICs following the implementation levels in the SOLAP architecture. Indeed, the conceptual expression of any IC depends on the concerned SDW concepts; when its physical translation depends on the SOLAP tier where it is implemented. In fact, he reveals that ICs can be specified using OCL and Spatial OCL at the conceptual level. However, Spatial OCL and UML do not allow specifying all IC proposed in that classification. This is related especially to metadata IC, the aggregation ICs specific to an application field and also visualization ICs. The author in [15], proposes an extension of the precedent classification by introducing a new class related to "Query ICs". This aims to avoid misinterpretations of the results as shown in Fig. 1.

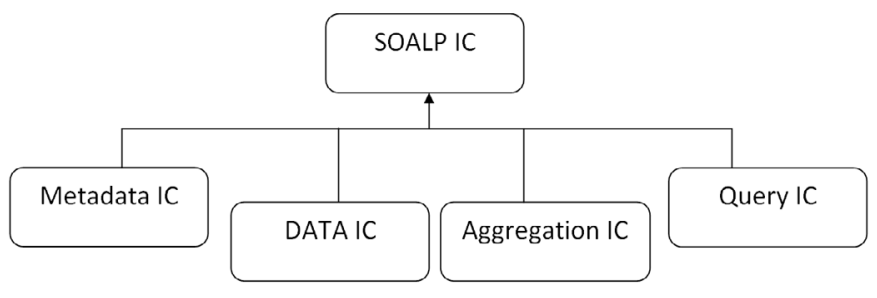

Fig. 1. Classification of SOLAP ICs.
However, the solution can be by adding in the UML profile:

1. more tagged values representing the main metadata,

2. more stereotypes allowing users to define their own aggregation ICs and also,

3. formalization of user profile for specifying visualization ICs according to their preferences.

\section{A. New facts ICs in Constellation Schema}

According to [13], a hyper-cell consists of a pair (L, MS) where L is a finite set of dimension levels and MS is a finite set of measures of a hypercube schema. The author classifies fact ICs into three categories. Indeed, a fact can be defined for one fact of a hyper-cell (f-lnter0), for several facts of the same hyper-cell (f-lnter1), and for several facts of several hyper-cells (f-lnter2) as shown in Fig. 2.

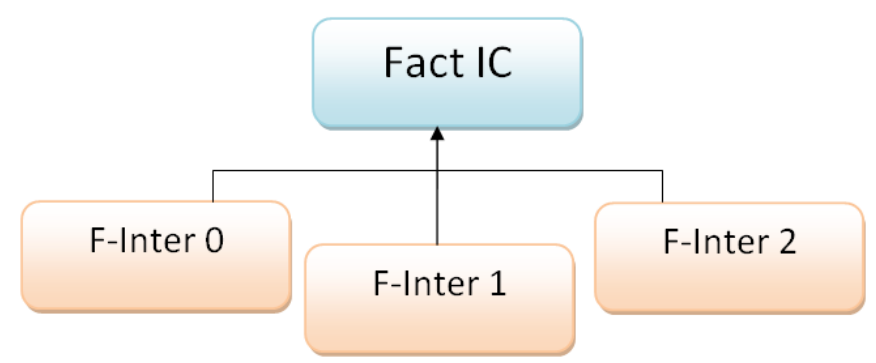

Fig. 2. Categories of fact ICs.

In this classification, the author considers ICs facts of one or several hyper-cells corresponding to the same facts table.

In our proposal, we consider new ICs for measures in several facts tables within the same hypercube as shown in Fig. 3. This situation can occurs when considering a constellation schema of a hypercube with several fact tables. In our case study, these fact tables are related to subjects of analysis in forestry management.

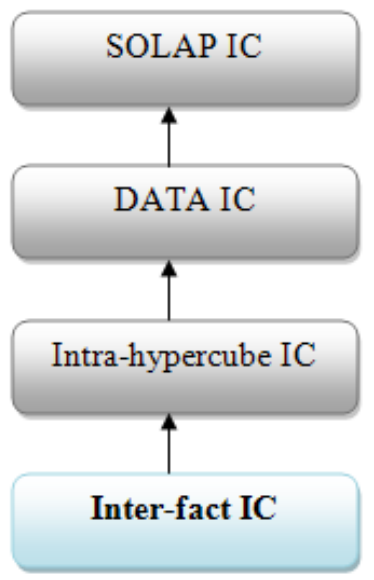

Fig. 3. Inter-fact ICs within a hypercube.

\section{B. Users ICs in SOLAP}

ICs related to the users of SOLAP system are numerous. In our proposal, when we use a hypercube with a constellation schema with several users, we classify these ICs into four categories as shown in Fig. 4. This classification may not to be exhaustive but we have focused on these parts that we consider directly linked to the user of such a system. 


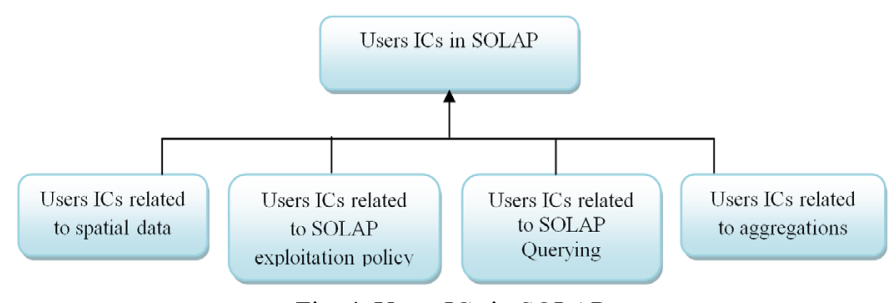

Fig. 4. Users ICs in SOLAP.

\section{1) Users ICs related to Spatial Data}

Integrity constraints apply to the database, a set of states that are valid by virtue of properties of objects that need to be stored according to every kind of final user of SOLAP system.

Geometric properties relationships of spatial data are related to «topological», «metric» and «ordering» relationships [30]. Here, semantic ICs concern the meaning of geographical features. The user can define integrity constraints which allow database consistency to be maintained according to his need [26].

After definition of the integrity constraints, we need to check if they are consistent. Indeed, before checking if a database satisfies a set of spatial semantic constraints, we need to make sure that the constraints themselves must not to be in conflict [31].

For example, a user rule must be activated to locate oil station at a given distance from the forest. That must not be in contradictory with another user-defined IC.

\section{2) Users ICs related to SOLAP Exploitation Policy}

At the modeling stage, the UML profile may be enriched with a stereotype called "UserProfile", which allows specifying constraints depending on particular information of a user or a group of users [32]. As a result, we customize information depending on the characteristics of the user that is requesting that information.

The Fig. 5. illustrates user ICs for an example of secure multidimensional modeling adapted from [32]. It shows a part of the Secure Model, named 'Hospital' which is based on a typical health-care system. In this example authors notice the following considerations related to the user of the system:

- Patients may access their own information as patients.

- deny access to data of patients who have been treated before the date of initial contract.

- deny access to admission information to users whose working area is different than the area of a particular admission instance.
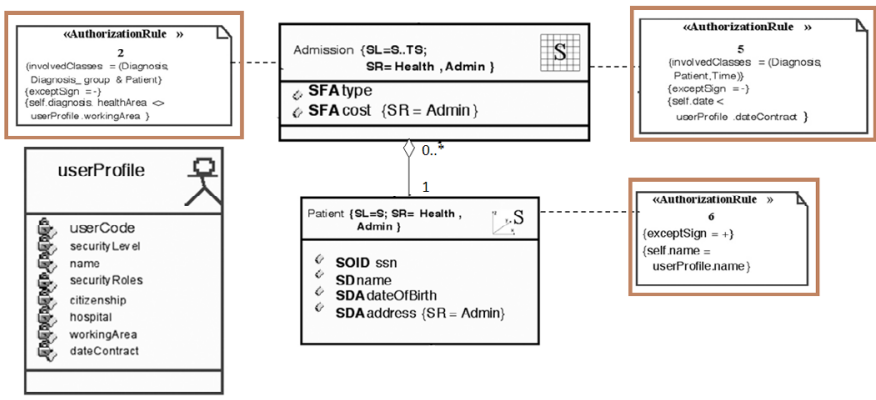

Fig. 5. Example of user ICs adapted from [32].

\section{3) Users ICs related to SOLAP Querying}

A SOLAP query is a combination of measures and members of different dimensions. A querying IC verifies the validity of this combination that may be effectuated by any user.
For example, it does not make sense to request geographic distribution of silviculturals effectuated by "Mostaganem" forest administration "conservation", in 1985 in "Zemoura" forest.

Here, according to the multidimensional model represented with a constellation schema proposed in Fig. 10, we have an analytical query which combines:

- Fact table: ["silviculturals"].

- SpatialMeasure: ["localisation"].

- Spatial aggregation level: [forest= "Zemoura"].

- Spatialaggregation level: [conservation= "Mostaganem"].

- Timedimension: [year="1985"].

The result will demonstrate that there is no silvicultural activities; while, according to the administrative division after February, 1984, "Zemoura" forest became under the direction of another conservation namely "Relizane" (western of Algeria). This can produce unreliable decision. So a query integrity constraint is able to prohibit this combination of parameters.

\section{4) Users ICs related to Aggregations}

The author in [15] has implemented aggregation ICs in the UML profile to avoid the implementation of semantically and structurally incorrect models of SDW.

For example, in order to force the user not to aggregate non-additive measures using the aggregate function "sum", the author defines the OCL statement which forbids this as shown in Fig. 6.

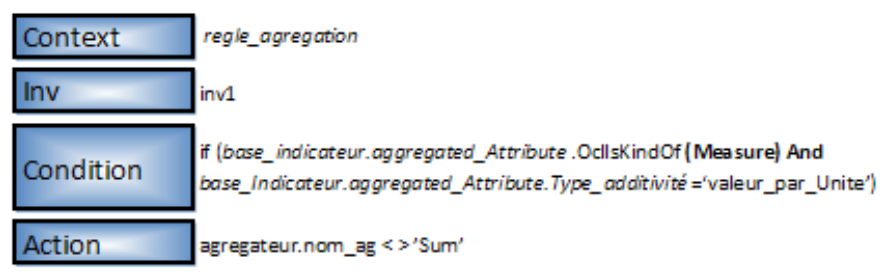

Fig. 6. Example of IC related to aggregations.

Generally, this kind of aggregation rules is valuable for every user. However, any user need to use a limit number of measures related to a specific facts table with group of dimension levels. Hence, we can attach the aggregation rules to the "UserProfile" stereotype defined in Case Study section.

\section{The Proposed System for User Integrity Constraints IN SOLAP "UIC-SOLAP"}

In the current study we propose a system namely "UIC-SOLAP" for checking integrity constraints which regulate the interactions between the user and the SOLAP process as shown in Fig. 7.

This system integrates all defined Integrity Constraints which are related to the users of SOLAP system along different stages of the latter. Indeed, in data sources which can be spatially referenced data, properties of objects may need to be stored according to every kind of final user.

In the interaction with SOLAP Server, the user uses combination of measures and members in analytical queries and he uses aggregation rules. These are concerned by the respect of Integrity Constraints.

Also, when using the user-interface of a SOLAP application, Integrity Constraints must be defined to respect particular information of a user, so that only concerned data is accessible. 


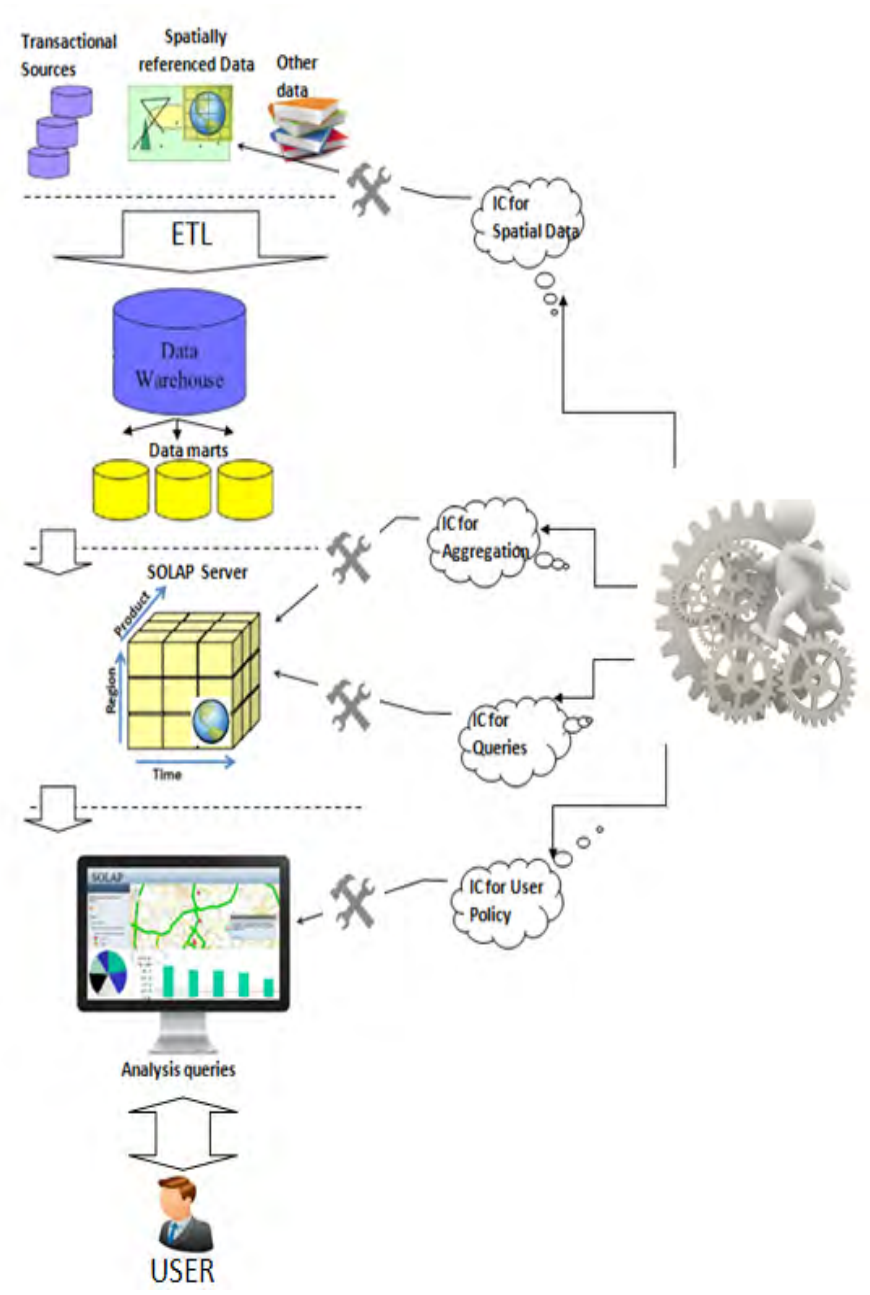

Fig. 7. "UIC-SOLAP" User Integrity Constraints in SOLAP.

\section{The Process Adopted By "UIC-SOLAP}

Throughout the SOLAP building and using, the process adopted by the proposed system namely "UIC-SOLAP", can be described as following:

- Firstly, the users can make different assertions in the spatial database according to their needs. Along this task, we must take care that these assertions are not in conflict between them.

- Secondly, to identify incorrect analytical queries, we must verify the validity of the combination of measures and members.

- Then, for defining correct aggregation rules, we must define in the modeling stage, stereotypes allowing users to define their own aggregation ICs.

- And finally, for the respect of user policy, formalization of user_profile concept is assured allowing specifying constraints depending on particular information of a user.

The operating algorithm for applying UIC_SOLAP can be as follows:

\author{
Algorithm \\ // check data user_integrity_constraints when analyzing \\ data, before applying "ETL" process \\ $S \leftarrow \emptyset ; / /$ set of spatial data properties \\ $P \rightarrow \emptyset$; // final list of properties initially empty \\ For (every kind of final_user of SOLAP) \\ Begin \\ S $\leftarrow$ Read (database_properties); //user will enter \\ //spatial properties according to him \\ Verify_contradictory $(P, S)$; //verify if enteredproperties \\ are contradictory with the others \\ While (not ok) // whiles is in contradictory with at \\ least one of database_properties \\ Begin \\ redefine_properties(S)// $S$ will be redefined \\ Verify_contradictory $(P, S)$; \\ End while \\ $P \leftarrow P+S$; // add $S$ tofinal list of properties \\ end for: \\ // (checking exploitation policy userintegrityconstraints) \\ When connecting to SOLAP \\ Read (user_id) \\ Apply_profile (user_id) //applying a set of parameters \\ allowing user to see only information related to his \\ specialty \\ // ... When querying the SOLAP \\ While (user is connected) \\ Begin \\ //checkingquerying user integrity constraints \\ Read(query) \\ Verify_combin (\{dimension_members\}, measure) \\ If (not OK) then show_message("inconsistent query will \\ generate inconsistent results"); \\ //checking querying userintegrityconstraints \\ Read (aggregation) \\ Verify_aggregation(query, aggregation_model) \\ //to Verify if requested aggregation function is \\ allowed to be applied \\ If (not OK) then show_message("not allowed aggregation \\ will generate inconsistent results")

\section{End while} \\ End Algorithm.
}

For the modeling, in our approach we use Unified Modeling Language (UML) since it becomes a standard using object-oriented concept and is widely used for designing various systems and software. UML can be also easily extended with profile to adapt it to specific domain namely multidimensional modeling.

The activity diagram of the proposed system namely "UIC-SOLAP" is shown in Fig. 8.

\section{VII.CASE StUdy}

The case study used to illustrate our proposal concerns the multidimensional (MD) analysis in a Spatial Decision Support System (SDSS) for forest management.

We use an extension of UML for MD modeling with three mechanisms: stereotypes, tagged values and constraints in the UML profile [33]. We use also Object Constraint language (OCL) to formalize IC constraints which refine the definitions of stereotypes and tagged values [34].

The forest SDW metamodel we propose is adapted from [35]. It 


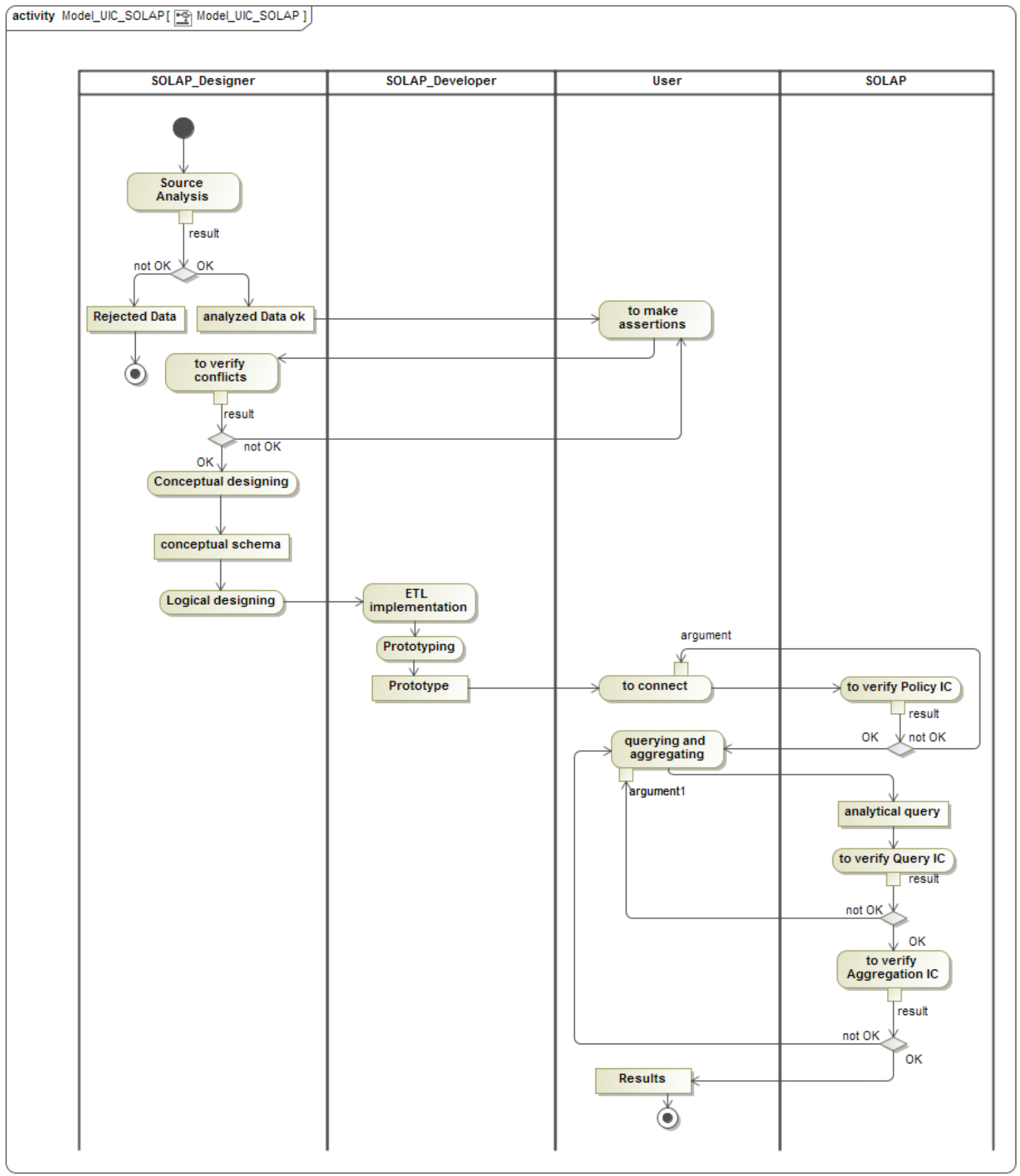

Fig. 8. Activity diagram for "UIC-SOLAP".

allows representing the main static concepts of the SDW. We used it thanks to its completeness and clarity. However, this metamodel does not allow creating a hypercube with several fact tables. So we introduce some modifications as depicted in Fig. 9. To do this we propose one-tomany cardinality in the composition relationship between hypercube package stereotype and the one of fact class. This allows having multiple fact tables within a hypercube [23]. Some stereotypes were removed and others were added where necessary.

We must ensure the modeling of all the measures, dimensions and hierarchies used for data aggregations required in the analysis process.

The class diagram for the forestry SDW is shown in Fig. 10. It is considered as spatial because it has at least one spatial measure and at least one spatial dimension.

In our case study, users are interested in the analysis of three aspects of the forestry domain, defined using a hypercube with three facts tables sharing some dimension tables in a constellation schema, which are:

1. «Silviculturals» facts table related to forestry activities such as afforestation, spacing, growth and yield, etc. It is described using three measures where one is spatial (localisation) and two are numerical (volume_fin and superficie);

2. «Event» facts table related to fires, illegal cut, etc. It is described using three measures where one is spatial (localisation) and two numerical which are volume_fin for the financial cost and superficie, the area concerned; 


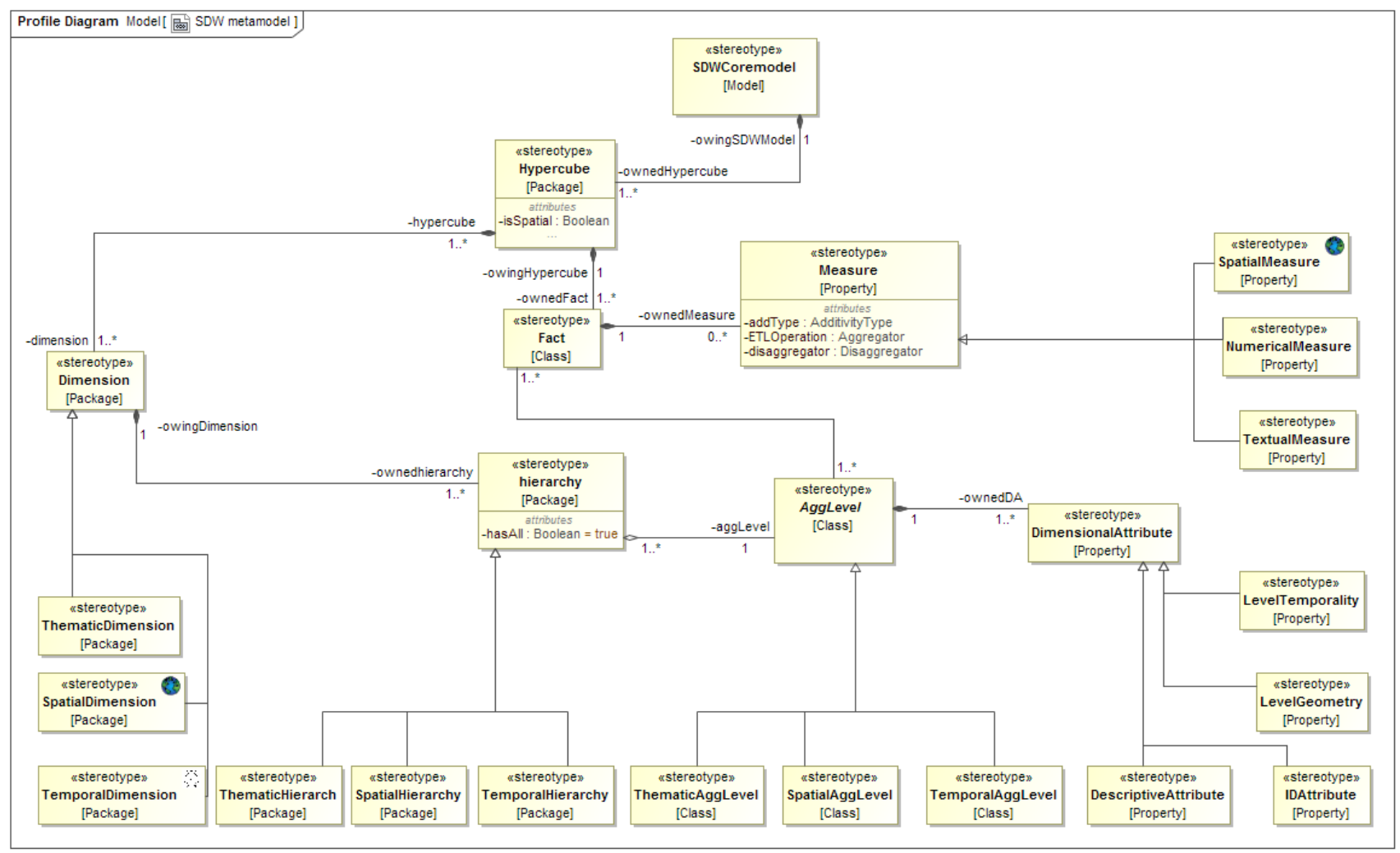

Fig. 9. Forest SDW metamodel.

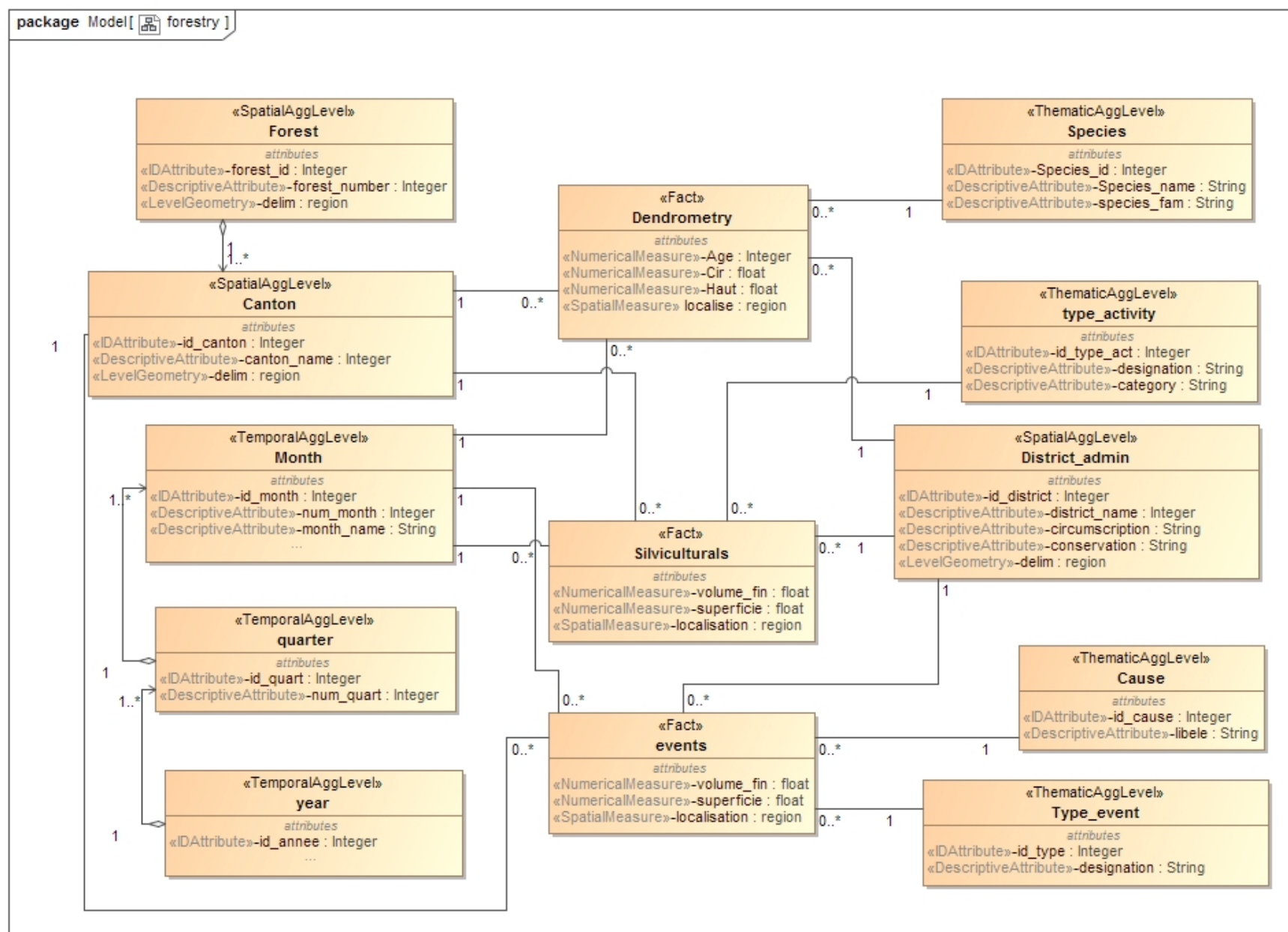

Fig. 10. Constellation schema of forest SDW. 
3. «Dendrometry» facts table related to the measurements of various characteristics of trees, such as their circumference, high, age, etc. It is described using three numerical measures (cir, haut, and age), and one spatial measure (localise).

Dimensions can be:

1. Temporal, to effectuate business analysis at three levels: month, quarter, or year;

2. Spatial, to effectuate business analysis at geo-referenced areas. We have two spatial dimensions: administrative with levels (district, circumscription and conservation); and ecologic with levels (canton and forest);

3. Thematic, which is related to the business processes. We have four thematic dimensions: Species, Type activity, Cause and Type_event.

We define the UserProfile stereotype for specifying IC depending on particular information of the user. In the class diagram, we have just one class of this stereotype; that has no association to other classes. It is used only for formulating IC as shown in Fig. 11.

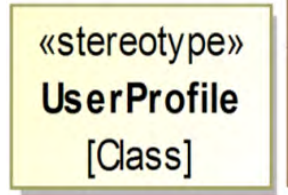

\begin{tabular}{|c|}
\hline \multicolumn{1}{|c|}{ "UserProfile» } \\
UserProfile
\end{tabular}

Fig. 11. UML Stereotype and Class for UserProfile.

For the new type of inter-facts IC within one hypercube cited in section 4.1, in the MD schema of forest SDW we introduce the assertion that verifies prospective conditions between two or more facts tables.

Fig. 12 shows an inter-facts IC between two facts tables Silviculturals and Events of the same hypercube. It specifies that superficies of reforestation must be greater than superficies destroyed by fires. We note that specification languages of ICs are explained in [13]. However, they go beyond the objective of the current study.

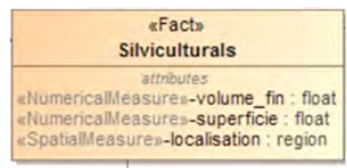

Superficies Silviculturals where type=reforestation $>$ Superficies Events where type_Events=Fires

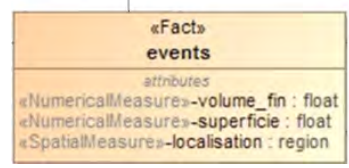

Fig. 12. Inter-facts IC.

\section{DisCUSSION OF RESULTS}

We have developed a SOLAP application with JAVA Eclipse, the object-oriented programming language. With the integration of ArcGis runtime SDK, the solution provides cartographic synchronization with tabular and diagram displays as shown in Fig. 13. Different users may pass their analytical queries using buttons and combo boxes available in the interface. They can also formulate their queries directly in MultiDimensional eXpression (MDX) language to communicate with the "Silvi" hypercube. The prototype is tested with a dataset concerning the Mediterranean forests of Mostaganem department situated in north western of Algeria.

Different users with different aspects of interest can manipulate the system with different manners. This can generate inconsistent analytical results. Hence, the formulation of ICs at different stages can avoid integrity problems.

In the following examples, we will focus on some ICs related to users of SOLAP application.

- Firstly, for the Users ICs related to spatial data, we take into consideration "user-defined" ICs. They are business rules which control the integrity of data stored and results. Here we have three examples related to users of each one of facts tables of the "Silvi" hypercube:

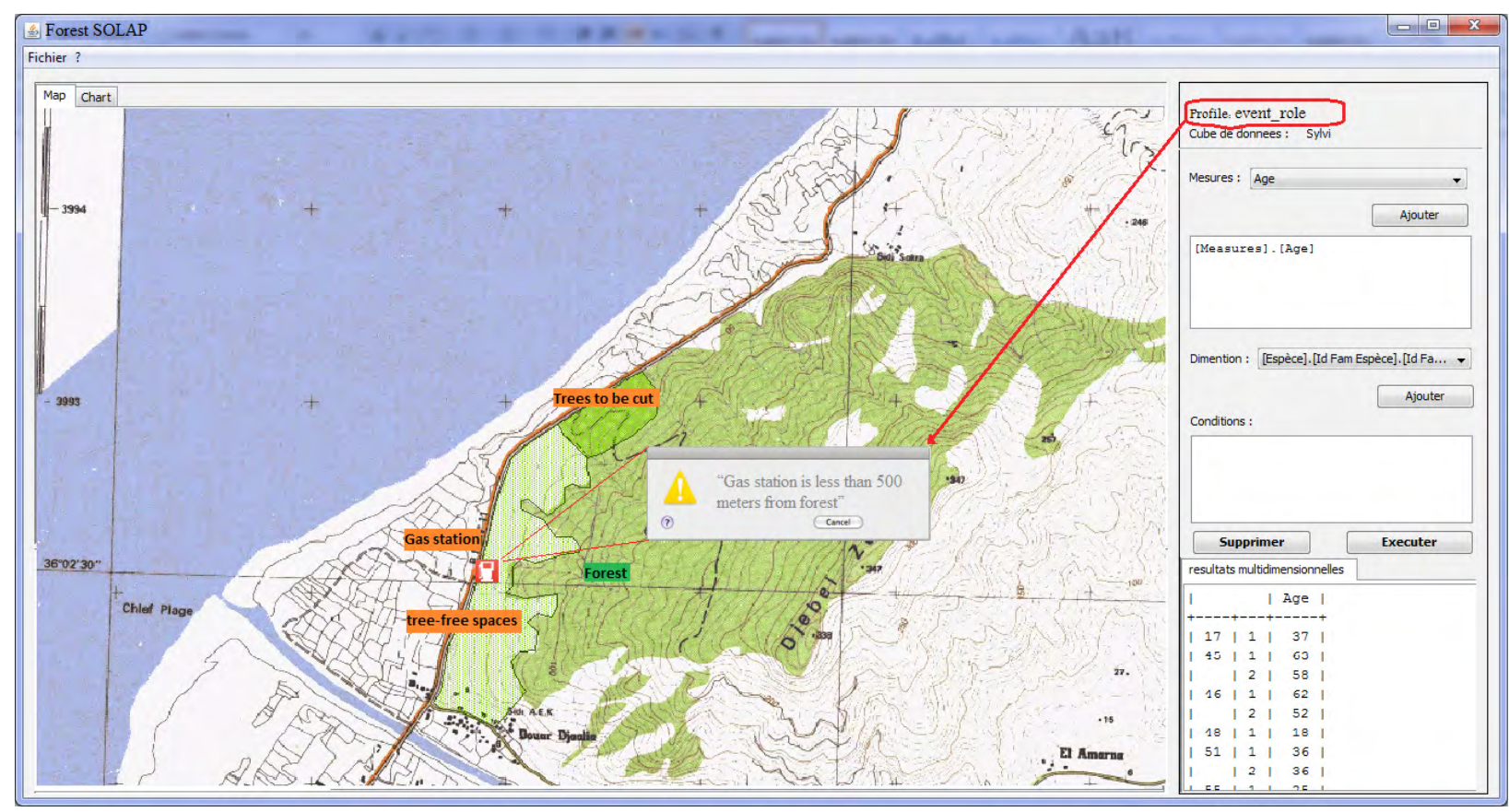

Fig. 13. Data User ICs in Forest SOLAP. 
1. "The distance between a forest and a gas station shall be more than 500 meters".

2. "Afforestation can be effectuated at forest spaces tree-free".

3. "it is authorized to cut down a tree when its circumference $>=$ $4 \mathrm{~m}$; height $>=20 \mathrm{~m}$; age $>=50$ and it is near a road".

These ICs may be contradictory since the users are heterogeneous and data will be used in deferent facts tables within the same hypercube.

In our proposal we try to remove these contradictory issues. Since our prototype may be used by any kind of users, they may query any one of facts tables. Their interests and rules may be different; hence, one user may not accept afforestation near a gas station even it is forest space tree-free; It can be dangerous for other spaces.

- For the Users ICs related to exploitation Policy, by the use of "UserProfile" concept, a "userrule" will be activated only if the concerned user is logging on. We create three profiles: Event_role, Silvicultural_role and dendrometry_role related to the users of Events, Silviculturals and dendrometry facts tables consecutive.

In this case, if the role is "Event_role" then only the related IC message appears "Gas station is less than 500meters from the forest"; despite "existing of very old trees near the road to be cut" and "existing of tree-free spaces within the forest to be reforested".

We note that the decisional problem can be solved by the application of collaborative group decision support system. However, it goes beyond the objective of the current study which is focused on user ICs in SOLAP application.

- For the Users ICs related to querying, considering the new administrative division, the system we propose verify the correctness of measures and dimension members combination in user queries. For the example above concerning combination of measures of Silviculturals facts table and ecological spatial dimension "forest=Zemoura" with administrative spatial dimension "conservation=Mostaganem" is false when time dimension member is after February 1984. Hence, the message in Fig. 14 appears.

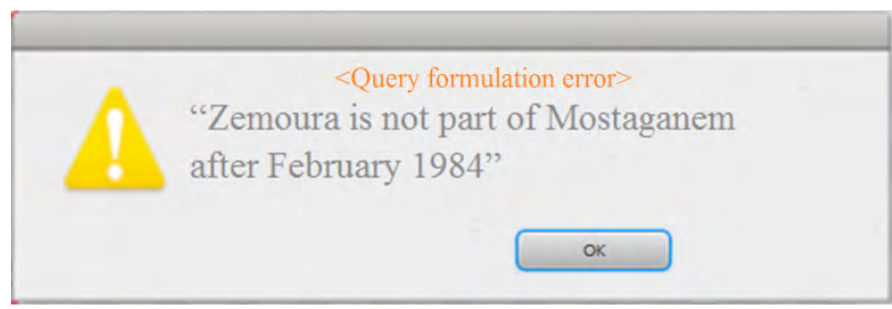

Fig. 14. Notification for non respected query IC

- And finally, for the Users IC related to aggregation, it defines how user wants to aggregate measures for calculating different indicators. Here, we indicate that every user has a set of indicators for the multidimensional analysis related to his specialty. So we propose that in the aggregation model to be defined in the UML profile, for each indicator we must specify which function to be used for aggregating the measure. ICs are defined in the metamodel and can be controlled when validating the conceptual model.

In this case, since the IC is related to the indicator and the latter is associated to the user, hence we can associate an IC including the 'UserProfile specialty' to the facts table's name. Indeed, this association can enrich the analysis consistency, since every user is able to effectuate correct queries relating to his specialty.

We note that querying a data warehouse is the job of the analyst or decision maker. However, in the current study we focus on the case when we use a hypercube with multiple facts tables. For example, in group decision systems, they may exist different actors (users). Each of them queries facts of his interest, and has no idea how to use other measures. This seems to be facultative, however it can be considered as a short cut and earlier control for measures aggregation. For example, concerning the dendrometry facts table, only users for whom Userprofile.specialty = "dendrometry", will be able to aggregating its measures.

\section{Conclusion and Future Work}

Spatial Data Warehouses (SDW) and SOLAP systems are used for discovering business information for Spatial Decision Support System (SDSS). In forestry management, the decision-making process requires the use of multidimensional model including multiple facts tables within the constellation schema.

As stated in studies presented in this paper, SOLAP analysis goodness depends on three quality aspects which concern data, aggregation and queries formulated. For addressing these issues, Integrity Constraints (ICs) have been acknowledged to be an excellent approach. Indeed, they define conditions that must be satisfied to improve the accuracy, consistency, and completeness of databases. ICs are often defined in conceptual models to allow handling these issues at the early stages of development.

The main objective of the current study is to highlight the ICs essentially related the users of SOLAP solution for the effective management of forestry areas and its activities. Indeed, we propose a UML modeling of the forest SDW profile. Next, we use it for the constellation schema modeling with three facts tables sharing spatial, temporal and thematic dimensions. Then, we propose a classification of ICs related the users of SOLAP systems. We propose also a new type of inter-facts ICs related to the use of hypercube with multiple facts tables. Finally, results of our proposal are shown using data concerning forest areas of Mostaganem in Algeria.

In our future works, we plan to extend our results by analyzing other interesting cases in which the SOLAP users ICs can be posed. This can be achieved by enriching our classification presented in this paper. We will address also, other aspects related to the quality in spatial data warehouses and verification of logical consistency in SOLAP Systems.

\section{ACKNOWLEDGMENT}

A part of this study was carried out in collaboration with experts from the Forestry Administration of Mostaganem (Algeria). Thereby we would like to acknowledge them with gratitude, in particular Mrs. "Touafek Narimann", the forest Conservator who left us forever and will remain in our hearts and our prayers as long as we live.

\section{REFERENCES}

[1] M.A.Mostafavi, G. Edwards and R. Jeansoulin, "An Ontology-based Method for Quality Assessment of Spatial Data Bases", Proceedings of the 4th International Symposium on Spatial Data Quality, Vienna, Austria. 2004.

[2] S. Vallières,J. Brodeur and D. Pilon, "Spatial Integrity Constraints: A Tool for Improving the Internal Quality of Spatial Data. Fundamentals of Spatial Data Quality, R. Devillers and R. Jeansoulin (Eds.), ISTE, pp. 161177. 2006.

[3] C. A. Hurtado, C. Gutierrez and A. O. Mendelzon, "Capturing summarizability with integrity constraints in OLAP", ACM Transactions on Database Systems, 30(3), pp. 854-886, 2005.

[4] F. Ghozzi, F. Ravat, O. Teste and G. Zurfluh, "Constraintes pour modèle et langage multidimensionnels». Ingénierie des Systèmes d'Information 9(1), pp. 9-34, 2004. 
[5] T. L. L. Siqueira, R. C. Mateus, R. R. Ciferri, V. C. Times, and C. D. A. Ciferri,"Querying vague spatial information in geographic data warehouses advancing geoinformation science for a changing world" Volume 1 of Lecture Notes in Geoinformation and Cartography, pp. 379397. Springer, 2011.

[6] D. Perez, M. J. Somodevilla, and I. H. Pineda,"Fuzzy spatial data warehouse : A multidimensional model", Decision Support Systems Advances, pp. 3-9. IEEE Computer Society, 2007.

[7] C. E. Dyreson, T. B. Pedersen, and C. S. Jensen, "Incomplete information in multidimensional databases", Multidimensional databases, pp. 282 309, IGI Publishing, 2003.

[8] K. Boulil, S. Bimonte and F. Pinet,»Un modèle UML et des contraintes OCL pour les entrepôts de données spatiales. De la représentation conceptuelle à l'implémentation», Ingénierie des Systèmes d'Information. 16(6): 11-39, 2011.

[9] F.Ghozzi, F. Ravat, O. Teste, and G. Zurfluh, "Modèle multidimensionnel à contraintes", Revue d'Intelligence Artificielle 17(1-3), 43-55, 2003.

[10] E. Malinowski and E. Zimanyi, "Advanced data warehouse design. Advanced Data Warehouse Design: From Conventional to Spatial and Temporal Applications", Data-Centric Systems and Applications, Volume. ISBN 978-3-540-74404-7. Springer, 2008.

[11] O. Glorio, and J. Trujillo,"An MDA Approach for the Development of Spatial Data Warehouses", In I.-Y. Song, J. Eder, et T. Nguyen (Eds.), Data Warehousing and Knowledge Discovery, Volume 5182 of Lecture Notes in Computer Science, pp. 23-32. Springer. 2008.

[12] J.-N.Mazon, J. Lechtenborger, and J. Trujillo, "A survey on summarizability issues in multidimensional modeling", Data \& Knowledge Engineering, 68(12), 1452-1469. 2009.

[13] M. Salehi, "Developing a model and a language to Identify and specify the integrity Constraints in spatial datacubes", $\mathrm{PhD}$ Thesis presented at the Faculty of high studies of LAVAL University, Quebec, 2009.

[14] F.Pinet and M. Schneider, “A unified object constraint model for designing and implementing multidimensional systems". In Journal on Data Semantics XIII, Volume 5530 of Lecture Notes in Computer Science, pp. 37-71. Springer. 2009.

[15] K.Boulil, "Une Approche Automatisée basée sur des Contraintes d'Intégrité définies en UML et OCL pour la Vérification de la Cohérence Logique dans les Systèmes SOLAP - Applications dans le domaine agri-environnemental", PHD thesis, University Blaise Pascal ClermontFerrand II, France, 2012.

[16] J. Ananda, "Implementing participatory decision making in forest planning", Environment Managing, 39, 534-544, 2007.

[17] A. Kangas, M. Kurttila, J. Kangas, T. Hujala and K. Eyvindson, "Decision Support for Forest Management”, Springer: New York, NY, USA, 2015.

[18] C. Prell, K. Hubacek and M. Reed, "Stakeholder analysis and social network analysis in natural resource management", Internet Society, 22(6), 501-518, 2009.

[19] E.Nordström, L.O. Eriksson and K.Öhman, "Integrating multiple criteria decision analysis in participatory forest planning: Experience from a case study in northern Sweden. Forest Policy Econ. 2010, 12, 562-574.

[20] M. Acosta and S. Corral, "Participatory Multi-Criteria Assessment of Forest Planning Policies in Conflicting Situations: The Case of Tenerife", Forests, 6, 3946-3969, 2015.

[21] N.Vainikainen, A.Kangas and J.Kangas, "Empirical study on voting power in participatory forest planning". Journal of Environmental Management, 88, 173-180, 2008.

[22] R. G. Crespo, V. H. M. García, J. J. R.Escribano, E. T. Franco andJ. M. C. Lovelle,"Forest fire detection and extinction software", In Proceedings of the 2009 International Conference on Artificial Intelligence, ICAI 2009 (Vol. 2, pp. 885-890).

[23] H.Sivakumar,C. Matt, M.Sethu, Z. Robert andG.Y.L. Denny, "Professional Microsoft SQL Server Analysis Services 2008 with MDX", Indianapolis, Indiana, USA. Wiley Publishing Inc Press.2009

[24] N.Stefanovic, J. Han, and K. Koperski,"Object-based selective materialization for efficient implementation of spatial data cubes",IEEE Transactions on Knowledge and Data Engineering, 12(6), 938-958,2000.

[25] Y. Bédard, S. Rivest and M.J. Proulx, "Spatial On-Line Analytical Processing (SOLAP): Concepts, Architectures and Solutions from a Geomatics Engineering Perspective. Data Warehouses and OLAP: Concepts, Architectures and Solutions", R. Wrembel and C. Koncilia
(Eds.), Idea Group Publishing, p. 298-319, 2007.

[26] S.Cockcroft, "A Taxonomy of Spatial Data Integrity Constraints", Geoinformatica, 1(4), pp. 327-343.1997.

[27] M.H. Bohlen, "Valid Time Integrity Constraints", Technical Report 94-30, Department of Computer Science, University of Arizona, Arizona, USA, 22 pages, 1994.

[28] K.Boulil, S. Bimonte and F. Pinet, "Un cadre conceptuel basé sur UML et Spatial OCL pour la définition des contraintes d'intégrité dans les systèmes SOLAP". Actes des 8èmes journées francophones sur les Entrepôts de Données et l'Analyse en ligne (EDA 2012). Bordeaux, France, 2012.

[29] M. Duboisset, Un système de contraintes d'intégrité OCL pour les bases de données Spatiales - Application à un Système d'Information pour l'Epandage Agricole, PHD thesis, école doctorale pour l'Ingénieur (SPI), Clermont-Ferrand, France, 2007.

[30] M.J. Egenhofer, "A FormaI Definition of Binary Topological Relationships", Proceedings of the 3rd International Conference on Foundation of Data Organization and Algorithms, LNCS 716, SpringerVerlag, Paris, France, p. 457-472,1989.

[31] L. Bravo, and M. A. Rodriguez, "Semantic Integrity Constraints for Spatial Databases", Proceedings of the 3rd Alberto Mendelzon International Workshop on Foundations of Data Management, Arequipa, Peru, May 1215, 2009.

[32] R. Villarroel, E. Fernández-Medina, M. Piattini and J. Trujillo, "A UML 2.0/OCL Extension for Designing Secure Data Warehouses", Journal of Research and Practice in Information Technology, 38(1), 2006.

[33] J. Trujillo, E. Soler, E. Fernández-Medina and M. Piattini, "A UML 2.0 profile to define security requirements for Data Warehouses", Computer Standards and Interfaces, 31(5), pp. 969-983, 2009.

[34] K. Boulil, F. Le Ber, S. Bimonte, C. Grag and F. Cernesson, "Multidimensional modeling and analysis of large and complex watercourse data: an OLAP-based solution", Ecological Informatics, 24, 90-106, 2014.

[35] K. Boulil, S. Bimonte, F. Pinet, "Conceptual model for spatial data cubes: A UML profile and its automatic implementation". Computer Standards \& Interfaces, 38, pp 113-132, 2015.

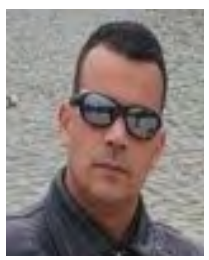

\section{Abdallah Bensalloua Charef}

Specialised in software engineering, Spatial Techniques, Geographical Information Systems (GIS), Remote Sensing and Geo-decisional Systems. He is computer science professor at the University of Mostaganem in Algeria.

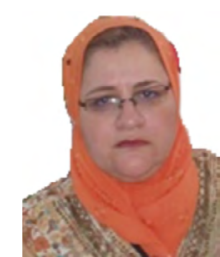

Hamdadou Djamila

Specialised in Decision Support Systems, Multicriteria Analysis and Collaborative and spatial decisional Systems. She is professor and leads a research team at the laboratory of computer science of Oran (LIO)at the University of Oran1 in Algeria. 